\title{
Image Adaptive Enhancement Strategy Based on Neural Network
}

\author{
Jiande Zhang*, Chenrong Huang \\ Department of Computer Engineering, \\ Nanjing Institute of Technology \\ Nanjing 211167, P.R.China \\ e-mail: zhangjiandexy@gmail.com
}

\author{
Jingui Lu \\ CAD Center \\ Nanjing University of Technology \\ Nanjing 210009, P.R.China \\ e-mail: njutcad@gmail.com
}

\begin{abstract}
Neural network algorithm is applied to image adaptive enhancement problem to determine the processing parameters in this paper. Based on the analysis of image enhancement theory, a BP neural network algorithm which contains forward propagation of information and back propagation of error is introduced to image filter designing, and the detailed computer implementation procedure is given for image enhancement. Numerical experiments have been performed to evaluate the efficiency of neural network algorithm for optimization problem of image enhancement.
\end{abstract}

Keywords-image enhancement; neural network; image filter; image processing

\section{INTRODUCTION}

Image enhancement is to improve the image quality by emphasizing some characteristics in image while restraining others selectively. The original image is usually processed to be a new form that the computer can understand easily [1]. Usually image enhancement technology can not make the image information increasing. Frequency domain algorithm, fuzzy processing and airspace law algorithm are the common methods in image enhancing. Fuzzy enhancement algorithm is the most popular method in image enhancing, and it can be divided to three steps: original image is processed to blurring data by image mapping technology, and then based on properties of characteristic plane, the fuzzy image data is processed, the processed information data can be mapped inversely to the spatial domain finally, and the enhanced image is obtained [2-4]. This algorithm makes good use of image inherent in the two justices, it is more suitable to human visual habits, but it requires manual intervention to determine the transition point and saturation point.

At present, many scholars have done many research works for image enhancement problem. Such as: waveletbased, curvelet-based, and contourlet-based algorithms have been used to improve the effect of image enhancement. An image enhancement method based on Laplacian pyramid has been researched by Greenspan [5-8]. BP (back-propagation) neural network algorithm has attracted a lot of attention due to its success in solving optimization and image processing problems. A BP neural network is one of the most maturely studied neural network, it has good self-learning, selfadapting, robustness and generalization ability, and a threelayered BP neural network can approach any non-linear functions with any precision[9-10]. Hence, there is a need to introduce BP neural network to improve the traditional image enhancement algorithm, and to achieve a better image processing effect.

This paper consists of four sections. The introduction is given in Section 1. The brief principles of BP neural network algorithm for image enhancement as well as the computer implementation procedure are presented in detail in Section 2. In Section 3 the experiments results using the method have been discussed in this paper to enhance images are given. Conclusions are addressed in Section 4.

\section{IMAGE ENHANCEMENT BY BP NEURAL NETWORK}

\section{A. Image Enhancement Strategy}

During image enhancing, usual methods can not carry out processing parameters automatically. To solve the problem, we consider a strategy to determine the parameters adaptively.

Generally, an original image can be divided into three parts: light and dark, gray concentrated in one area. To image enhancement, there are usually four typical contrast transform curves, we can made use of the normalized incomplete Beta function to automatically fitting image enhancement of the four kinds of curves. The Beta function can be defined as:

$$
\mathrm{F}(\mu)=\mathrm{Y}^{-1}(\alpha, \beta) \int_{0}^{\mu} t^{\alpha-1}(1-t)^{\beta-1} d t
$$

Where, $\alpha>0$ and $\beta<10, \mathrm{Y}(\alpha, \beta)$ can be described as:

$$
Y(\alpha, \beta)=\int_{0}^{1} t^{\alpha-1}(1-t)^{\beta-1} d t
$$

To the different values of $\alpha$ and $\beta$, normalized incomplete Beta function has a different transform curves.

In this paper, BP neural network is used to determine the best image transform function adaptively. Original image is normalized firstly, and based on this, the image is transformed by nonlinear function, then the optimal parameter values of the transform function can be carried out by BP neural network adaptively, the enhanced image is got after reverse normalization finally. In this processing, BP neural network is simply a gradient descent method designed to minimize the total error (or mean error) of the output computed by the network, and there is an input layer, an output layer, and one or more hidden layers between them.

Suppose the original image is $\varphi(x, y)$, and the enhanced image is $\varphi^{\prime}(x, y)$, and $(\mathrm{x}, \mathrm{y}) \in \Omega$ is defined domain of image. After the normalized transformation, gray 
values of image are between $[0,1]$, the process is described as:

$$
g(x, y)=\left[\varphi(x, y)-L_{\min }\right] /\left[L_{\max }-L_{\min }\right]
$$

where $L_{\max }$ is the maximum gray value, $L_{\min } L_{\max }$ is the minimum gray value. The nonlinear transformation function is $\mathrm{F}(\mu)$, the transformation process is:

$$
g^{\prime}(x, y)=F(g(x, y))
$$

Finally, the reverse normalization process is:

$$
\varphi^{\prime}(x, y)=255 g^{\prime}(x, y)
$$

\section{B. Computer Implementation of the Strategy}

The strategy discussed above can be applied to the image enhancement problem. During enhancing image, we consider using BP neural network to optimize the two Beta parameters, and then the normalized incomplete Beta function is applied to enhance images. The process includes: image normalization, Beta parameters initialization, BP neural network training, image reverse transformation and image enhancement. The procedure is shown as figure 1 .

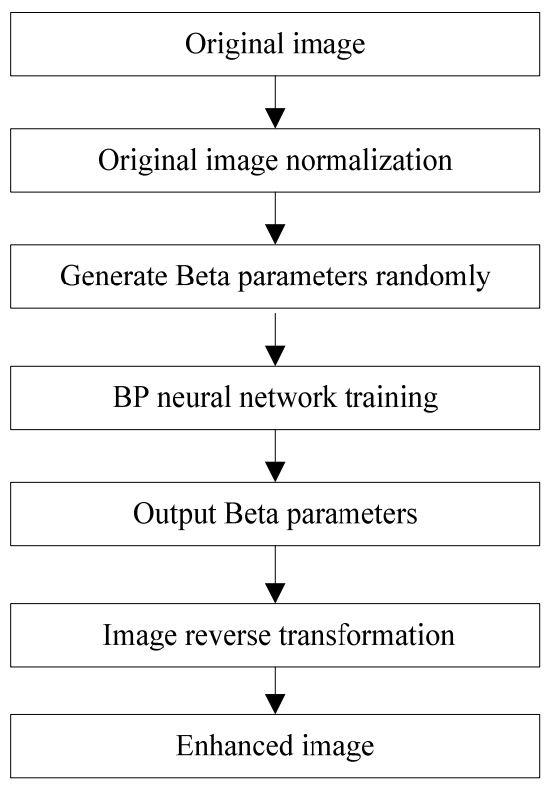

Figure 1. Flowchart of image enhancement

From figure 1, the image enhancement procedure can be described as follows:

Step 1. Read the original image and save the row, column and greyscale values of each pixel;

Step 2. Normalization of the original image: Greyscale value of each pixel is transformed between $[0,1]$ by the above formula (3);

Step 3. Generate two Beta parameters randomly which values is between $[1,10]$. The initial parameters of Beta function are rarely more than 10 but always no less than 1 by experiences;
Step 4. Training Beta parameters by BP neural network. BP Neural Network originates from BP algorithm which contains two parts: forward propagation of information and back propagation of error. Through forward propagation of information and back propagation of error the weights are constantly adjusted between neurons to reduce the error between the expected output and the actual output achieving the purpose of training the samples. During the determining of the two parameters, we can use a three - layer BP neural network taking effect in training the two input variables, the output is the two optimal Beta parameters. The energy function is constructed by image contrast which can be described as:

$$
f(g)=\frac{1}{h w} \sum_{x=1}^{h} \sum_{y=1}^{w} g^{2}(x, y)-\left[\frac{1}{h w} \sum_{x=1}^{h} \sum_{y=1}^{w} g(x, y)\right]^{2}
$$

Where, $g$ is the image, $g(x, y)$ is the gray value of one pixel point in image $\mathrm{g}, \mathrm{h}$ is the height of image, $\mathrm{w}$ is the width of image ;

Step 5. Image reverse transformation. The reverse transformation process can use the formula (4) and (5);

Step 6. Based on the two parameters determined by BP neural network, the image can be transformed by the normalized incomplete Beta function finally.

\section{APPliCATION OF THE STRATEGY}

Numeral experiments are taken out to verify the electiveness of the discussed method by applying it to a digital mage - the standard Lenna image. The image experimented in this paper is shown in figure 2, image histogram of the original Lenna image is shown in the figure 3 , and the enhanced image is shown in figure 4, image histogram of the enhanced image is shown in the figure 5. The original image is of size $256 * 256$ pixels, and is 8 bits/pixel, gray-scale. The result of experiment is shown as follow:

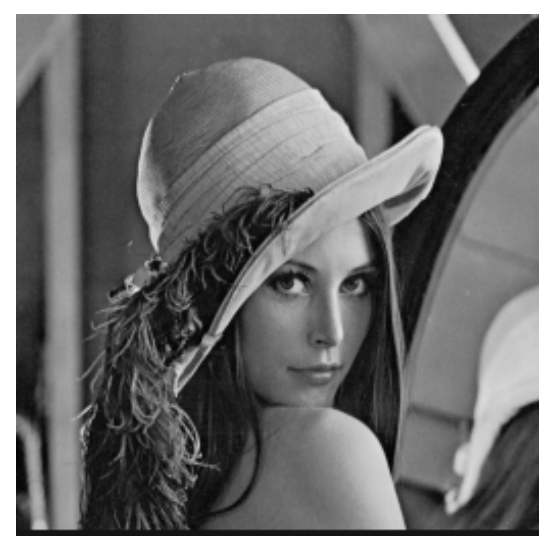

Figure 2. Original image for enhancement 


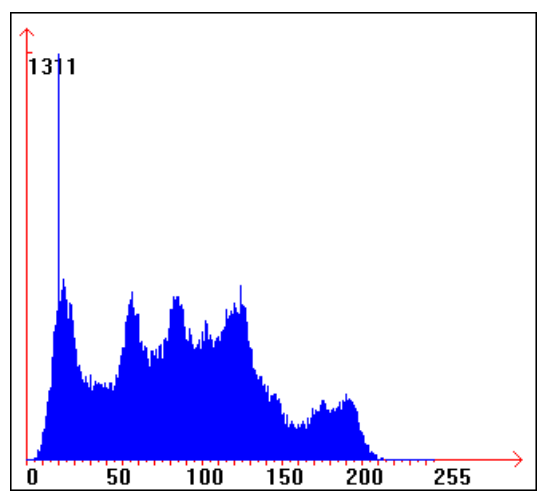

Figure 3. Image histogram of original image.

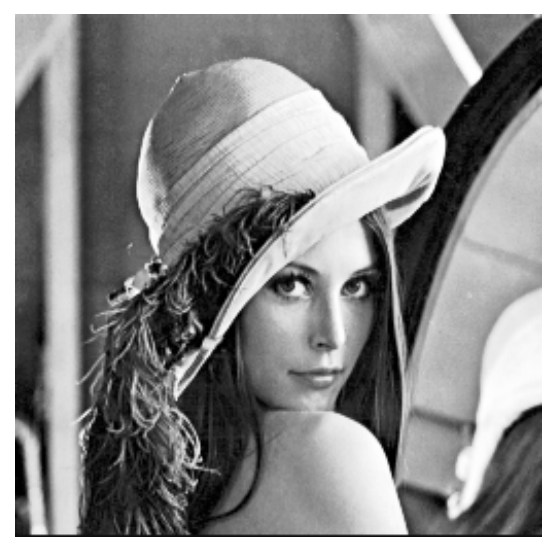

Figure 4. Enhanced image.

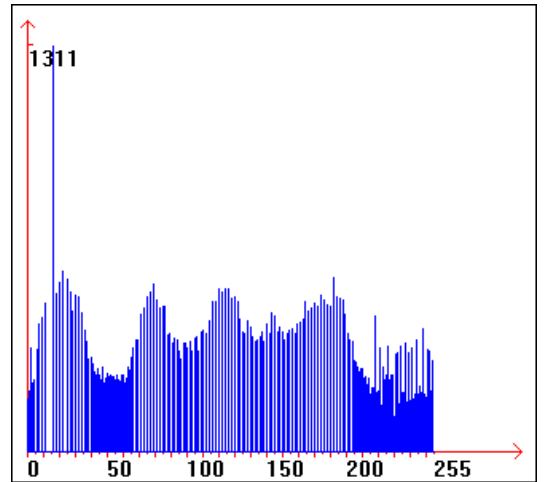

Figure 5. Image of enhanced image

From the experiment result, we can see that: in the original image, the face and hat texture detail is not very obvious and the image is a bit of dark, the contrast in the image is also lower. While, when the image has been enhanced by the algorithm discussed in this paper, the above problems have been solved and we obtain a satisfactory images, which is brightness and contrast are well balanced, visual effects of the experiment image has been improved very well. For the given image, when we use BP neural network to compute the transformation parameters, the parameters value respectively is 3.65 and 8.12 . Meanwhile, from the histogram figure 3 and figure 5 , it is obviously that the image gray distribution is more even and the dark gray region allocation in the image is more reasonable.

\section{ACKNOWLEDGMENT}

This work is supported in part by the Scientific Research Foundation of Nanjing Institute of Technology (Grant No.CXY201110). We are grateful to express our thanks. We are grateful for the computing resources provided by colleagues in College of Computer Engineering and CAD Certer of Nanjing University of Technology.

\section{REFERENCES}

[1] X.Luo,J.Tian, "Image Enhancement and Minutia Matching Algorithms in Automated Fingerprint Identification System ," Journal of Software,vol.13,May. 2002, pp. 946-956, doi: 1000-9825/2002/13 (05)0946-11.

[2] N.Zhang, "Research on Image Enhancement Technology," Computer Simulation,vol.24,Jan. 2007, pp. 192-195, doi: 1006 - 9348( 2007) 01 - 0192- 04.

[3] Z.Yang,J.Zhou,X.Yan,M.Huang, "Image Enhancement Based on Fractional Differentials," Journal of Computer-Aided Design \& Computer Graphics,vol.20,Mar. 2008, pp. 343-348, doi: 1005 2312( 2008) 07 - 2198- 03.

[4] Z.Liu,W.Yang,Q.Zhu, "A Comparative Study on Several Algorithms of Image Enhancement," Journal of Hefei Normal University,vol.28,Dec. 2010, pp. 60-63, doi: 1674-2273(2010)060060-04.

[5] W.Mao,X.Zhao, "Enhancement algorithm for low2illumination color image based on neural network," OPTICAL TECHNIQUE,vol.36,Mar. 2010, pp. 225-228, doi: 10021582(2010)02-0225-04.

[6] R.Wang,X.Zhang,S.Shuai, "Image Enhancement in the Compressed Domain Based on Retinex Theory," Journal of Computer Research and Development,vol.48,Dec. 2011, pp. 259-270, doi: 1000 - 1239P CN 11 - 1777 P T P

[7] C.Gao,J.Zhou, "Image Enhancement Based on Quaternion Fractional Directional Differentiation ," ACTA AUTOMATICA SINICA,vol.37,Feb. 2011, pp. 150-159, doi: 10.3724/SP.J. 1004.2011.00150.

[8] L.Liang,W.He,L.Lei,W.Zhang,H.Wang, "Survey on enhancement methods for non-uniform illumination image ," Application Research of Computers,vol.27,May. 2010, pp. 60-63, doi: 1674-2273(2010)060060-04.

[9] W.Deng,Q.Zheng,L.Chen,X.Xu, "Research on Extreme Learning of Neural Networks," Chinese Journal of Computers,vol.33,Feb. 2010 , pp. 279-287, doi: 10. 3724/ SP. J . 1016. 2010. 00279.

[10] S.Li,L.Liu,Y.Xie, "Chaotic prediction for short-term traffic fow of optimized BP neural network based on genetic algorithm ," Control and Decision,vol.26,Oct. 2011, pp. 1581-1585, doi: 1001-0920 (2011) 10-1581-05. 